\title{
« Simplifier l'orthographe, oui, mais... ». Attentes, réserves et ambivalences dans les discours d'enseignant.e.s sur ce que serait une « bonne » réforme de l’orthographe française
}

\author{
Groupe RO \\ (Rectifications Orthographiques)
}

\section{Introduction}

Selon les points de vue, l'orthographe française est source de difficultés, d'insécurité, de fierté ou encore de bien d'autres sentiments ou attitudes que l'on pourrait qualifier d'ambivalents... Elle est également, en raison même de cette ambivalence des attitudes à son égard, l'objet de débats récurrents sur le fait de savoir, entre autres, s'il serait opportun ou non la changer, de la réformer, de la simplifier... Ces débats, souvent passionnés et relativement peu informés linguistiquement et sociolinguistiquement, n'ont pas réellement permis aux décideurs et instances en charge des politiques linguistiques de prendre des décisions claires et lisibles et acceptables par le plus grand nombre. Les atermoiements et flottements dans l'application des rectifications de 1990 nous semblent en être un symptôme.

Se saisissant de la question de savoir si, à l'heure actuelle, les usagers du français, au-delà de la seule communauté des francophones de France, seraient favorables à un aménagement de l'orthographe, un groupe de chercheurs issus de différents pays (Groupe RO, 2011) a entrepris d'évaluer l'existence d'une telle demande de réforme de la norme graphique du français.

L'enquête par questionnaires, menée auprès d'enseignants et de futurs enseignants de français, montre qu'à des degrés divers selon les pays et le statut professionnel des sujets, une majorité est favorable à une rationalisation du système graphique du français ${ }^{1}$. Dans le cadre de cette contribution nous ne nous intéresserons qu'à un aspect parmi les nombreux qui ont été abordés dans le questionnaire, en l'occurrence à ce que les sujets interrogés considèreraient comme une «bonne réforme ».

Après avoir rappelé quelques éléments de méthodologie générale et particulièrement la façon dont nous avons procédé pour réaliser l'analyse thématique des réponses, nous décrirons à grands traits les principaux thèmes et leur importance relative. Puis nous nous attacherons à montrer qu'au-delà des réponses portant sur telle ou telle condition ou modalité jugée souhaitable ou au contraire indésirable par les répondant.e.s, il ressort de leurs discours une polarisation autour de deux attitudes : l'une que nous considérons comme pragmatique et l'autre comme manifestant un attachement aux aspects symboliques de l'orthographe.

\footnotetext{
${ }^{1}$ Pour un aperçu exhaustif des résultats, nous renvoyons à Moreau \& Dister (2012).
} 


\section{Méthodologie générale de l'enquête et question $F$}

\subsection{L'enquête}

Une enquête par questionnaire a été menée auprès d'un peu plus de 2000 témoins francophones ressortissants tant de pays du «nord »(Belgique, Canada, Suisse, France) que du «sud»(Algérie, Maroc). Étant donné leur position privilégiée dans les processus de transmission de la norme orthographique, on a jugé prioritaire d'interroger, d'une part, des enseignant.e.s de français, aux niveaux primaire et secondaire et, d'autre part, des étudiant.e.s se destinant à devenir enseignant.e.s. Au total ces deux catégories totalisent 1738 répondant.e.s.

Le questionnaire, qui comportait 68 questions fermées (échelles de 4 à 6 degrés) et 8 questions ouvertes, a majoritairement été renseigné par les témoins sous sa forme écrite, et dans quelques cas sous forme électronique.

Si l'un des premiers objectifs de la recherche entreprise était d'évaluer l'existence d'une demande sociale en matière de réforme de l'orthographe, il s'agissait également de tenter de déterminer la nature de cette demande, et notamment les contours de ce que les enquêtés considèrent comme une "bonne réforme ». Il s'agissait donc d'explorer selon quelles modalités une action d'aménagement de l'orthographe pourrait être recevable pour ces scripteurs un peu particuliers que sont les enseignants et les futurs enseignants, généralistes dans l'enseignement primaire ou spécialistes du français dans le secondaire.

Le questionnaire comportait plusieurs questions conçues pour tenter de cerner tout à la fois :

- ce que les participants à l'enquête considèrent comme objets/domaines de l'écriture à réformer ;

- si ces lieux de réformes sont à leur sens possibles ou souhaitables ;

- quelles sont les modalités de décision et d'application d'une réforme ;

- et s'il existe selon eux un certain nombre d'effets souhaitables ou indésirables d'une telle action de politique linguistique.

Nous nous intéresserons ici à une des questions ouvertes de ce questionnaire (la question F).

\subsection{La question $F$}

Cette question était libellée de la façon suivante : «À votre avis quelles conditions devrait remplir une BONNE réforme de l'orthographe? »

Les lexèmes choisis pour inviter les témoins à donner leur avis (conditions, et bonne) étaient peu contraignants et autorisaient donc des réponses d'orientations diverses. Effectivement, les réponses proposent différents types de conditions évaluées à l'aune d'une multitude de paramètres : certaines sont plutôt focalisées sur les objets/domaines d'une réforme, sur ses orientations pragmatiques (rendre accessible ou faciliter 
l'apprentissage) ou idéologiques ${ }^{2}$ (qui ne nuisent pas à la richesse de la langue française), sur ses modalités de décisions (concertation) ou de mise en œuvre (immédiate et inconditionnelle vs précédée par une phase transitoire). Nous avons donc dégagé des ensembles de propositions qui renvoient aux différentes interprétations des termes conditions et «bonne réforme » donnés en consigne.

La question $\mathrm{F}$ était placée plutôt en fin de questionnaire, après deux séries de propositions (questions 9 à 36 et 37 à $53^{3}$ ) explorant d'une part les représentations ou attitudes à l'égard de l'orthographe, de ses fonctions et effets linguistiques, symboliques, sociaux, et d'autre part, leurs sentiments concernant la modification potentielle de différents points d'orthographe. Pour ces deux séries de questions fermées, les témoins devaient estimer leur degré d'adhésion ou de rejet sur une échelle de 1 à 6 . On ne peut exclure qu'en mettant en 'phrases' des arguments pro- ou antiréformistes, en exposant les enquêtés à des énoncés préconstruits à dominantes logiques, axiologiques, idéologiques..., en passant en revue différentes possibilités de changement, les items qui précédaient la question $F$ aient pu influencer certaines réponses. En témoigne par exemple cette déclaration d'une enseignante de primaire québécoise : «Je n'y crois pas... plus j'avance dans le questionnaire, plus je suis contre une réforme. »

\subsection{Codage des réponses}

Avant de traiter des réponses exprimées et de la façon dont elles ont été exploitées, il convient de souligner que le nombre de non réponses (codées DM) est assez considérable.

a) Plus d'un quart des sujets n'énonce aucune condition

Comme on peut le constater dans le tableau ci-dessous, le pourcentage moyen de DM pour l'ensemble des pays est de $28,4 \%$.

\section{Tableau 1: Proportion de non-réponses à la question $F$ en valeur absolue et pourcentage}

\begin{tabular}{|l|l|l|l|l|l|l|l|}
\hline & Algérie & Maroc & Belgique & France & Québec & Suisse & Tous pays \\
\hline
\end{tabular}

\footnotetext{
${ }^{2}$ Nous entendons ici idéologie au sens d'idéologies langagières telles que définies dans la tradition de l'anthropologie linguistique nord-américaine anglophone, comme par exemple par Silverstein (1979: 193) : «[...] les idéologies de la langue [ou du langage], ou idéologies linguistiques / langagières, sont tout un ensemble de croyances à propos de la langue et du langage telles que formulées par les utilisateurs comme une rationalisation ou une justification de la manière dont ils perçoivent la structure d'une langue/du langage et son usage » (notre traduction). On peut d'ailleurs compléter cette première définition par ces précisions de Woolard $(1998: 3):$ « [...] les idéologies de la langue/du langage ne concernent pas seulement la langue/le langage. Elles considèrent et mettent en œuvre des liens entre la langue et l'identité, l'esthétique, la moralité et l'épistémologie. A travers de tels liens, elles sous-tendent non seulement les formes et les usages linguistiques, mais également la notion même de personne et de groupe social, en même temps que des institutions sociales fondamentales telles que les rituels religieux, la socialisation des enfants, les relations de genre, l'État-nation, l'École, et la Loi ».

${ }^{3}$ Les réponses à ces questions sont analysées dans plusieurs contributions dans Moreau \& Dister (2012).
} 


\begin{tabular}{|l|c|c|c|c|c|c|c|}
\hline Nombre de sujets & 119 & 94 & 374 & 748 & 196 & 207 & 1738 \\
\hline Nombre de DM & 44 & 31 & 79 & 266 & 31 & 43 & 494 \\
\hline \% de DM & $37,0 \%$ & $33,0 \%$ & $21,1 \%$ & $35,6 \%$ & $15,8 \%$ & $20,8 \%$ & $28,4 \%$ \\
\hline
\end{tabular}

Comme on peut également le voir dans le Tableau 1, les pourcentages de témoins n'ayant pas répondu à la question $\mathrm{F}$ oscillent entre un minimum de $15,8 \%$ pour le Québec et un maximum de $37 \%$ pour l'Algérie, suivie de près par la France $(35,6 \%)$. Nous serons amenés à revenir sur la signification éventuelle de ces nombreuses nonréponses.

\section{b) Propositions de conditions et regroupements thématiques}

A partir d'une analyse de contenu des réponses écrites, nous avons codé des segments de texte correspondant à ce que nous avons considéré comme des « propositions élémentaires ». Au total, nous avons identifié et codé 1903 items, chaque réponse contenant souvent plusieurs propositions. Prenons l'exemple de cette réponse fournie par une enseignante du primaire québécoise.

Chaque nouvelle règle ne devrait pas comporter d'exception. Une liste complète et précise des modifications devrait être donnée à chaque enseignant et accessible sur internet pour tous. L'application devrait être imposée à une date fixe.

Nous y avons isolé quatre propositions :

Chaque nouvelle règle ne devrait [pas comporter d'exception] (codée 19)

Une liste complète et précise des modifications devrait être donnée [à chaque enseignant] (codée 13)

et [accessible sur internet pour tous] (codée 14)

[L'application devrait être imposée à une date fixe] (codée 9)

En procédant de la sorte, nous avons obtenu 28 catégories, que nous avons listées dans la deuxième colonne du Tableau 2.

Nous avons ensuite procédé à des regroupements de propositions en ensembles présentant une cohérence thématique, repris dans la première colonne du Tableau 2. Ces blocs thématiques sont au nombre de cinq, auxquels s'ajoute une catégorie résiduelle (« Autres »).

Tableau 2 : Codage en propositions et regroupements en thèmes retenus pour l'analyse

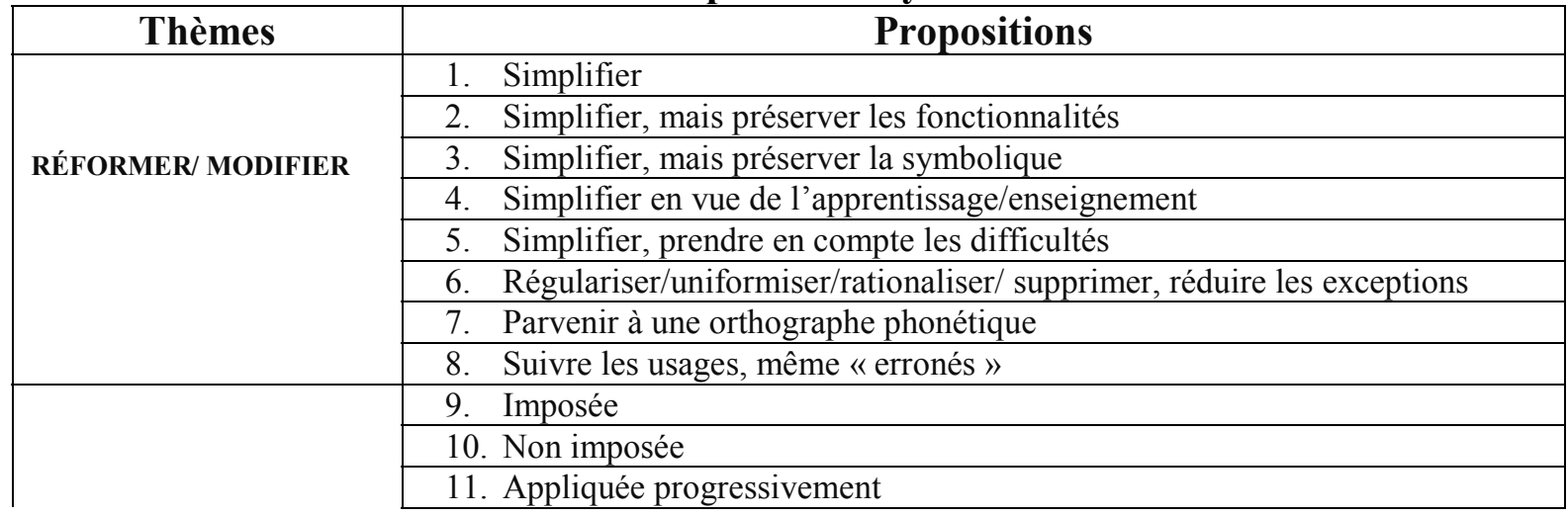




\begin{tabular}{|c|c|}
\hline \multirow{5}{*}{$\begin{array}{l}\text { MODALITÉS } \\
\text { D'APPLICATION } \\
\text { ET DE DIFFUSION : } \\
\text { Pour être bonne, une } \\
\text { réforme doit être : }\end{array}$} & 12. Appliquée immédiatement \\
\hline & 13. Diffusée par la formation des professionnels \\
\hline & $\begin{array}{l}\text { 14. Diffusée par information et explication à la population (via les médias } \\
\text { notamment) }\end{array}$ \\
\hline & 15. Diffusée par l'enseignement aux élèves \\
\hline & $\begin{array}{l}\text { 16. Appliquée, acceptée dans l'usage (notamment utilisateurs légitimes et } \\
\text { ouvrages de réf) dans la francophonie internationalisation }\end{array}$ \\
\hline \multirow{6}{*}{$\begin{array}{l}\text { QUALITÉS : } \\
\text { Pour être bonne, une } \\
\text { réforme doit : }\end{array}$} & 17. Etre simple/accessible (par le plus grand nombre) \\
\hline & 18. Être claire \\
\hline & 19. Être logique (cohérente, systématique, ne pas compliquer) \\
\hline & 20. Rendre l'orthographe, la langue accessibles, être utile, pratique, faciliter \\
\hline & 21. Être radicale/définitive \\
\hline & $\begin{array}{l}\text { 22. Ne pas dévaloriser la langue (être de portée limitée, ne pas dénaturer, } \\
\text { niveler par le bas, pas de sms, d'écriture phonétique, etc.) }\end{array}$ \\
\hline \multirow[t]{2}{*}{ 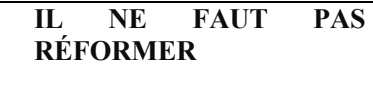 } & 23. Il n'y a pas de bonne réforme \\
\hline & 24. Ne pas réformer, mais enseigner plus ou mieux \\
\hline \multirow{3}{*}{$\begin{array}{l}\text { MODALITÉS } \\
\text { d'ÉLABORATION/ } \\
\text { ADOPTION : Pour être } \\
\text { bonne, la réforme doit }\end{array}$} & 25. Respecter certaines modalités de décision/adoption \\
\hline & 26. Prendre en compte l'hétérogénéité de la francophonie \\
\hline & $\begin{array}{l}\text { 27. Être prise après consultation de la population (acceptée dans le principe); } \\
\text { consensuelle }\end{array}$ \\
\hline AUTRES & 28. Divers \\
\hline
\end{tabular}

- Réformer/modifier. Dans la première catégorie (propositions 1 à 8), nous avons inclus les items qui touchent l'objet même d'une réforme, objet qui peut être condensé par les termes simplifier ou modifier,

A mon avis, la bonne réforme c'est de trouver une règle d'orthographe claire qui ne contient pas de cas particuliers ; exemple "s" dans le pluriel (EtuSec ALG) ${ }^{4}$

Cet ensemble thématique regroupe 8 propositions qui totalisent 716 mentions, soit 37,6\% des 1903 propositions énoncées : autrement dit, 41,2\% des sujets se prononcent en faveur de modifications/simplifications orthographiques plus ou moins radicales.

Simplifier les règles, avoir moins d'exceptions (EtuPri BEL)

Écrire ce que l'on prononce comme en espagnol et en italien. Le français se veut des origines latines pourquoi ne pas faire comme nos voisins latins (EtuPri FRA)

Garder du sens ; simplifier réellement sans créer de nouvelles exceptions (cf 1991); aller vers ce qu'écrit la majorité (EtuPri FRA)

Les propositions faites en ce sens sont parfois assorties de précisions de type simplifier pour, ou de restrictions sur le mode simplifier mais :

Une réforme qui ne soit pas réductrice, qui tienne compte de la richesse de la langue française tout en la simplifiant (EtuPri FRA)

\footnotetext{
${ }^{4}$ Etu $=$ étudiant se destinant au métier d'enseignant, Pro = enseignant en poste $;$ Pri $=$ primaire $;$ Sec $=$ secondaire $; \mathrm{ALG}=$ Algérie $; \mathrm{BEL}=$ Belgique $; \mathrm{FRA}=$ France $; \mathrm{MAR}=$ Maroc $; \mathrm{QUE}=$ Québec $; \mathrm{SUI}=$ Suisse.
} 
Réduire le nombre de graphies possible pour le même son, tout en gardant la graphie qui permet de décoder le sens d'un mot par dérivation (la famille à laquelle il appartient) (ProSec QUE)

Simplifier l'orthographe sans trop changer le sens... (EtuPri QUE)

Faciliter l'écriture sans pour autant nier l'origine des mots et ne pas "bêtifier" la langue (EtuPri BEL)

Enlever certains aspects complexes et plus ou moins justifiés. Exemples...

- Hôpital vs Hopital : qu'apporte l'accent circonflexe? Réforme

- Cote vs Côte : l'accent circonflexe distingue par la prononciation deux termes différents : Statu quo (ProSec QUE)

Une analyse qualitative approfondie des structures oppositives ou concessives devra être menée, mais on peut d'ores et déjà remarquer que ces restrictions portent sur un ensemble très disparate et qu'elles semblent manifester une ambivalence des scripteurs. En effet, le nombre élevé de ces structures semble indiquer que, d'un côté, les sujets ont conscience des difficultés objectives et vraisemblablement vécues (par eux-mêmes ou leurs élèves), tandis que, de l'autre, ils manifestent un attachement, qui peut paraitre paradoxal, aux formes orthographiques traditionnelles.

Selon moi, une "bonne" réforme devrait faciliter l'apprentissage de l'orthographe, mais en la facilitant, en la simplifiant, l'orthographe perdrait de sa valeur (EtuPri FRA)

Supprimer ce qui complique mais ne pas enlever ce qui fait la particularité de certains mots (EtuPri SUI)

Ne pas changer les beautés de la langue française, mais être plus tolérante sur les erreurs, quand la règle est compliquée (EtuPri SUI)

- Modalités d'application. Le deuxième bloc thématique (propositions 9 à 16) est constitué de propositions qui concernent les modalités d'application d'une éventuelle action sur l'orthographe (doit-elle être imposée ou non, d'application immédiate ou différée, brusque ou progressive ?) et, de façon non négligeable, le « périmètre » et les voies de diffusion :

Elle devrait être appliquée en priorité par les enseignants. Cependant, elle ne devrait pas être imposée mais enseignée en priorité. La population devra l'appliquer au cours du temps (délais) (EtuPri FRA)

Les 8 items de ce bloc thématique rassemblent 378 occurrences, qui représentent une proposition sur cinq $(19,9 \%)$ et qui sont exprimées par un peu plus de $21 \%$ des témoins.

- Qualités. L'ensemble suivant (17-22) agrège des propositions qui portent de façon prioritaire sur les qualités qu'une réforme devrait présenter pour être bonne. Ces qualités peuvent tenir à la présence de certaines caractéristiques (ainsi tel futur enseignant du primaire français juge qu'une bonne réforme devrait être «définitive, simple, claire, stimulante, passionnante») ou, au contraire, à l'absence de caractéristiques jugées indésirables :

Etre logique et applicable dans tous les cas; ne pas modifier profondément la "silhouette" de la langue française (pas d'écriture texto, la possibilité de comprendre la langue française d'"avant la réforme" (EtuPri FRA) 
On dénombre 451 occurrences des propositions énonçant une qualité que devrait posséder une réforme pour être bonne. $\mathrm{Si}$ on isole, au sein de ce groupe thématique, la proposition 22 qui exprime une volonté de voir limitée la portée d'une éventuelle réforme (160 occurrences pour cette seule proposition sur laquelle nous reviendrons), les items 17 à 21 recueillent 291 mentions, soit 16,7\% des témoins ou $15,3 \%$ du total des items.

- Ne pas réformer. Deux types de propositions expriment une hostilité plus ou moins radicale vis-à-vis d'une réforme (propositions codées 23 et 24). Si la question invitait explicitement à énoncer ce que seraient les conditions pour qu'une réforme soit bonne, certains enquêtés ont en quelque sorte profité de l'espace mis à leur disposition pour rappeler qu'ils étaient opposés à l'idée de toute réforme. Certains témoins, hostiles à l'idée de réformer l'orthographe, ont répondu, en argumentant parfois, qu' " aucune réforme ne serait bonne »:

Je ne pense pas qu'une réforme de l'orthographe soit le fond du problème (EtuPri FRA)

Pour moi, l'orthographe n'a pas besoin d'être réformée (EtuPri BEL)

Je pense qu'une réforme de l'orthographe est inutile. Celle de 1990 n'a déjà servi à rien. En Allemagne, depuis la dernière réforme, les personnes ne savent plus s'il faut écrire un $\beta$ ou ss suivant les mots. Ça devient n'importe quoi, pour ceux qui savent (EtuPri FRA)

D'autres, peut-être plus conscients des difficultés des élèves, proposent (reprenant une des questions fermées) de ne pas réformer l'orthographe, mais de mieux l'enseigner :

Il faut l'enseigner bien (EtuSec ALG)

Pour moi, il n'y a pas besoin de réforme. Il faudrait seulement insister beaucoup plus sur l'apprentissage de la langue à l'école primaire! A mon avis, c'est lamentable de vouloir ainsi dénaturer la langue française (EtuPri FRA)

Ces deux types d'opinion sont exprimés à 147 reprises $(7,7 \%$ des 1903 propositions recueillies), par $8,5 \%$ des sujets, score nettement plus faible que celui obtenu par les propositions appelant une simplification de leurs vœux.

On ne peut exclure que d'autres sujets aient pu exprimer le même type d'attitude d'opposition en ne répondant pas à la question $\mathrm{F}$. Mais si les non-réponses sont nombreuses, il est peu probable qu'elles aient toutes cette signification pour tous les sujets. Ainsi, certains ont pu s'abstenir de répondre parce que n'ayant pas d'opinion sur la question, ou pressés d'en terminer, etc.

- Modalités d'élaboration et/ou d'adoption. Nous avons regroupé dans une quatrième catégorie les propositions qui thématisent la façon dont une réforme de l'orthographe devrait être élaborée et adoptée (propositions 25, 26 et 27).

Parmi les conditions que devrait remplir une bonne réforme de l'orthographe c'est la collaboration avec tous les pays francophones (EtuSec MAR)

Prendre en compte l'opinion de la population et de tous les pays francophones (EtuPri QUE)

Faire voter les enseignants et les étudiants en enseignement (EtuPri QUE) 
- $\quad$ Autres. Cette catégorie est constituée des propositions que nous n'avons su ou pu classer dans une des autres catégories.

Non compétente en la matière (EtuPri FRA)

Ne pas intégrer les anglicismes! = Réformer ce qui n'apporte pas grand chose (tel les points cités avant que j'ai relevés) (EtuPri SUI)

Au final, 102 propositions ont été classées dans cette catégorie "Autres », soit $5,3 \%$ des propositions formulées par les répondants ${ }^{5}$.

A partir de ce codage des contenus propositionnels, nous avons dans un premier temps essayé de dégager des tendances fortes, qui se manifestent quels que soient l'origine géographique et le statut des témoins. Nous avons ensuite comparé les résultats par pays et par catégories de répondants.

Les tendances chiffrées présentées ci-après décrivent les pourcentages de réponses contenant un segment de discours codé dans l'une des 28 catégories par rapport à l'ensemble des propositions exprimées et codées. Ainsi, le pourcentage de propositions 6 , qui est égal à $10,1 \%$, correspond à 193 segments de réponse comportant une proposition que nous avons codée 6, sur un total de 1903 propositions exprimées et codées.

\section{Résultats : les grandes tendances}

Pour observer les grandes tendances dans les discours obtenus à la question ouverte F, il convient d'opérer des regroupements des propositions présentées dans le tableau cidessus. Un premier regroupement peut être fait, celui des propositions qui, d'une façon générale, jugent opportune une simplification orthographique - propositions 1 à 6 , parmi lesquelles 3 et 6 sont reliées thématiquement respectivement aux propositions 19 et 22, comme nous l'approfondirons dans le paragraphe suivant.

\subsection{Pas de réforme radicale, mais des simplifications en tous genres...}

En tout premier lieu, il faut souligner que 43,5\% des propositions faites par les personnes interrogées évoquent l'idée, certes avec des nuances que nous détaillerons par la suite, de simplifier l'orthographe. Cette question de la simplification est donc assez centrale, ce qui l'est moins, c'est la question de savoir quoi simplifier.

\section{Elle devrait régulariser les règles de grammaire UNIQUEMENT (EtuSec BEL)}

Elle doit simplifier les règles comprenant beaucoup d'exceptions comme le pluriel des adjectifs composés par exemple (EtuPri FRA)

Elle ne devrait modifier que les mots dont la graphie est " arbitraire" (par exemple, les consonnes doubles qu'on ne distingue pas à l'oral, les «fausses lettres étymologiques) (EtuPri QUE)

Il s'agit souvent de régulariser les exceptions, ce qui chez certains sujets n'est d'ailleurs pas senti comme une réforme :

\footnotetext{
${ }^{5}$ Les propositions classées «Autres » lors d'un premier codage individuel ont, pour une partie d'entre elles, été recodées (alimentant par exemple les catégories 25, 26).
} 
Pas de réforme, juste diminuer le nombre d'exceptions (EtuPri FRA)

Une bonne réforme de l'orthographe c'est simplifier ses règles (EtuSec ALG)

La crainte est souvent exprimée qu'une réforme n'ajoute encore des exceptions :

- garder du sens - simplifier réellement sans créer de nouvelles exceptions (cf 1991) - aller vers ce qu'écrit la majorité. (EtuPri FRA)

ou qu'elle rende la reconnaissance des mots trop difficile :

Elle ne devrait pas proposer de modifications trop " radicales ", qui changent trop la forme globale du mot (par exemple, " métrize » pour « maîtrise ») (EtuPri QUE)

On le voit, si la question de la simplification semble importante, les contours de cette simplification sont assez flous et rien ne fait vraiment consensus. Une seule chose est sure, c'est que le spectre d'une orthographe " phonétique » ou d'une " écriture SMS » en effraie plus d'un et que, parmi les personnes qui ont répondu à la question, il s'en trouve seulement 36 , soit $1,8 \%$, qui réclament une réforme radicale allant dans le sens d'une application systématique du principe phonographique (proposition 7).

Quant aux buts de cette simplification, il s'agit le plus souvent, de la part des enseignants et futurs enseignants, de rendre l'orthographe accessible au plus grand nombre en en facilitant l'apprentissage (proposition 4 reliée à 20).

Faciliter l'apprentissage de l'O. < Faire progresser les élèves dans ce domaine. Amoindrir son importance par rapport à d'autres apprentissages plus essentiels. <Simplifier son utilisation pour la rendre accessible à tous (EtuPri FRA) (codé 4 et 20)

\subsection{Deux attitudes contrastées}

Si l'on regarde les premiers résultats généraux, qui présentent les pourcentages moyens des différentes propositions rapportés au nombre total de propositions, en ne tenant pas compte, dans un premier temps, des variables indépendantes, on remarque que les contenus propositionnels qui concentrent le plus grand nombre de réponses sont ceux que nous avons codés $3,6,19$ et 22 et qui correspondent schématiquement à deux grandes tendances face à une éventuelle réforme de l'orthographe.

Figure 1: pourcentage de mentions de chaque proposition (toutes variables confondues) 


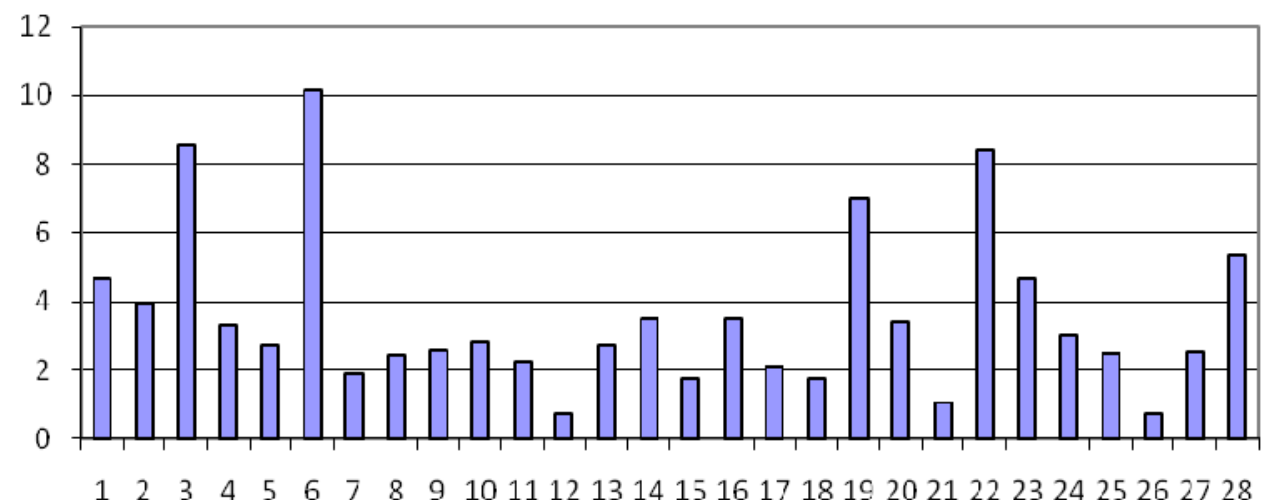

Ces deux tendances peuvent selon nous renvoyer à deux attitudes différenciées, de façon quasi polaire: la première renverrait plutôt à un attachement aux aspects symboliques de l'orthographe (histoire et étymologie, beauté, richesse, etc.), tandis que la seconde pourrait être qualifiée d' " attitude à tendance pragmatique », plus résolument ancrée en synchronie et visant à régulariser l'écriture du français, sans doute pour qu'elle soit plus facile d'utilisation pour le plus grand nombre.

\section{a) Attachement aux aspects symboliques}

Cette tendance est la plus importante. Elle s'exprime au travers des propositions 3 «Simplifier l'orthographe, mais conserver» tout ce qui est de l'ordre de l'idéologie orthographique, telle que définie plus haut - et 22 - «Ne pas dévaloriser la langue »et manifeste un attachement à une certaine tradition orthographique. Toute atteinte aux formes orthographiques - qu'elle soit d'ordre grammatical ou lexical - attenterait aussi à l'esthétique, à l'histoire et au patrimoine.

Elle devrait se limiter à un certain nombre de sujets sensibles et donc ne pas "arroser" tout azimut. Elle devrait également ne pas perdre de vue les racines de la langue. (ProCol SUI)

Ne pas toucher aux mots qui ont des raisons historiques ou sociales de s'écrire de cette façon. (EtuSec QUE)

Une bonne réforme ne doit pas être effectuée dans le but de rendre l'orthographe complètement différente de ce qu'elle a été jusqu'à aujourd 'hui. L'histoire de l'écriture française et ses origines pourraient être perdues si on adoptait une réforme qui applique les points mentionnés ci-haut. Il y a une raison pour que le mot costume, par exemple, s'écrive ainsi. Rebâtir l'orthographe en changeant tous les acquis représente, pour moi, tout le contraire d'une bonne réforme. Si le but d'une réforme de l'orthographe est de rendre l'écriture accessible à tous, il faut s'attarder à la façon dont l'orthographe s'enseigne dans les écoles. Il faut accorder plus de temps de lecture aux étudiants afin qu'ils se familiarisent avec l'orthographe. (EtuPri QUE)

Pour un certain nombre de sujets, comme pour ce dernier témoin, ce n'est pas l'orthographe qu'il faut réformer, mais son enseignement :

(...) En ce sens, ce n'est pas l'orthographe qui devrait être réformée, mais son enseignement (...) (EtuPri FRA) 
Je ne voudrais pas d'une réforme de l'orthographe... C'est trop facile, je trouve, d'appliquer le principe de "c'est trop compliqué, donc on simplifie !" Les règles orthographiques sont une question d'étude et il n'y a pas de raison que cela change. (EtuPri BEL)

Simplifier les méthodes d'enseignement de l'orthographe (EtuSec MAR)

On notera que cet attachement aux valeurs symboliques de l'orthographe est évoqué par $4,7 \%$ des personnes qui ont répondu à la question $(3,3 \%$ de l'ensemble de l'échantillon).

La moyenne des réponses fournies par les témoins aux questions 5 et 7, qui portent sur le positionnement par rapport à une réforme, permet de distinguer deux groupes : l'un, qu'on appellera les DEF, dont la moyenne est inférieure à 3,5, se montre hostile à un changement de normes; pour l'autre groupe, les FAV, la moyenne est supérieure à 3,5 : leur position est plutôt favorable à une réforme. Les propositions 3 et 22 émanent davantage de personnes du groupe DEF comme le montre l'histogramme ci-dessous ${ }^{6}$.

Figure 2 : propositions mentionnées selon l'attitude plutôt favorable ou défavorable à une réforme (en \%)

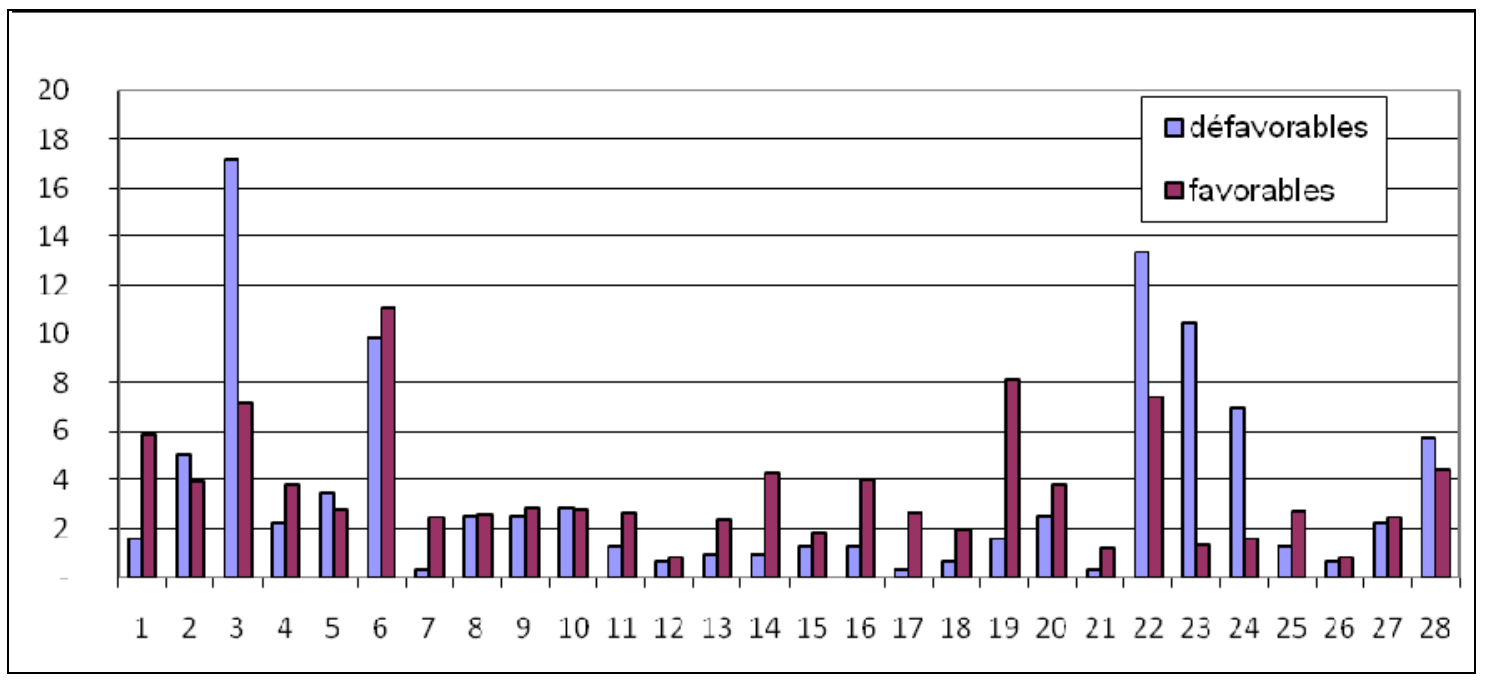

Cependant, la proposition 3 est l'une de celles pour lesquelles l'opposition entre les pays du Nord (Belgique, France, Québec, Suisse) et ceux du Sud (Maroc, Algérie) est statistiquement significative (dans toutes les comparaisons par paires, le test de Fischer indique des valeurs $\mathrm{p}<0,01)$. Cette proposition n'est en effet jamais évoquée dans les pays du Sud comme le montre l'histogramme ci-dessous, établi sur les pourcentages de mentions.

\footnotetext{
${ }^{6}$ Les légères distorsions de moyennes entre les graphes sont dues au fait que les données manquantes se répartissent inégalement sur les différentes questions.
} 
Figure 3 : opposition Nord / Sud sur la proposition 3 (en \%)

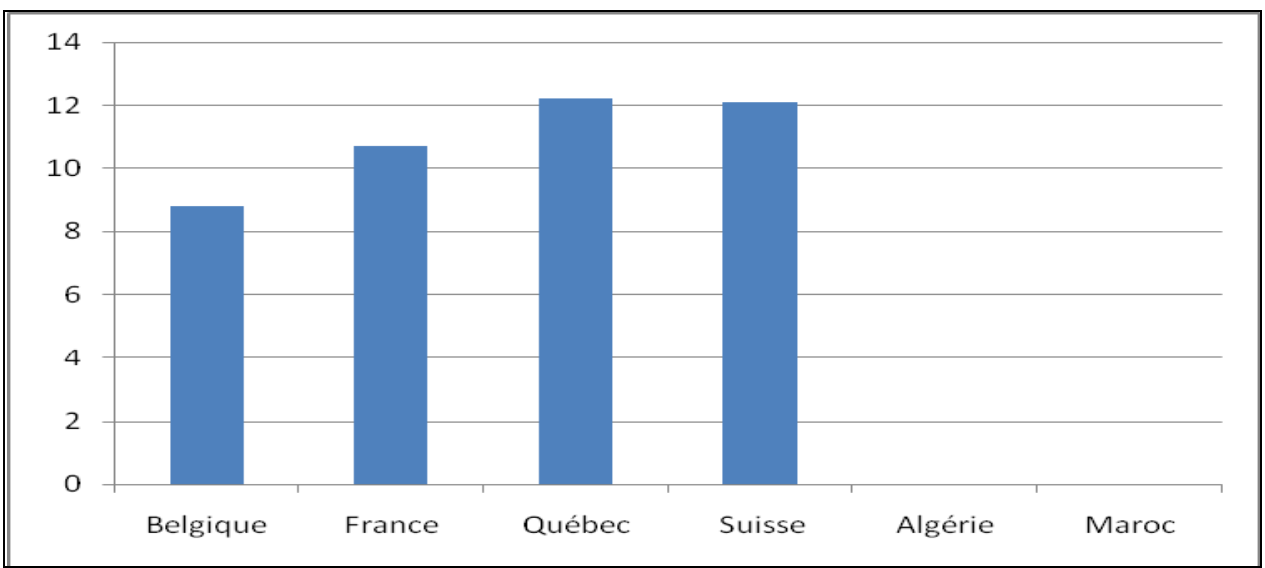

Il semblerait donc que les facteurs d'attachement symbolique aux formes orthographiques du français en vigueur sont moins prégnants dans les deux pays du sud. Mais le français pouvant y être considéré comme une langue seconde, l'attachement idéologique à la langue se portent plutôt sur l'arabe littéraire Fus-ha.

\section{b) Tendance pragmatique}

La tendance pragmatique s'exprime, quant à elle, dans les propositions 6 ( «égulariser / rationaliser ») et 19 (« Être logique, cohérente »). Elle est, spécialement pour la proposition 19, plutôt le fait de personnes favorables à une réforme, comme on peut l'observer plus haut dans la figure 2 .

Il s'agit pour les sujets de conserver l'essentiel de l'orthographe tout en la rationalisant, en la débarrassant de certaines exceptions, en l'allégeant de certaines zones de difficultés perçues comme trop complexes (les participes passés sont souvent cités).

Le but est de faciliter la forme écrite de la langue en réduisant les illogismes et les pièges inutiles. (ProPri QUE)

Une bonne réforme de l'orthographe devrait éliminer les aberrations. Il ne s'agit pas de simplifier pour rendre la langue plus facile, mais bien de la rendre plus logique (ProPri QUE)

Réduire le nombre d'exceptions, simplifier, organiser logiquement (EtuSec QUE)

La logique doit être à la base de la réforme. (EtuPri FRA)

Réforme qui simplifie, supprime, un certain nombre d'exceptions qui compliquent l'apprentissage de la LF mais qui respecte le sens de certaines orthographies, leur origine et qui permet une activité réflexive sur la langue française (EtuPri FRA)

Cette dimension référant à la logique tisse un réseau d'oppositions significatives entre les différents pays comme le montre l'histogramme suivant (Figure 4).

Figure 4 : Répartition dans les différents pays de la proposition 19 (en \%) 


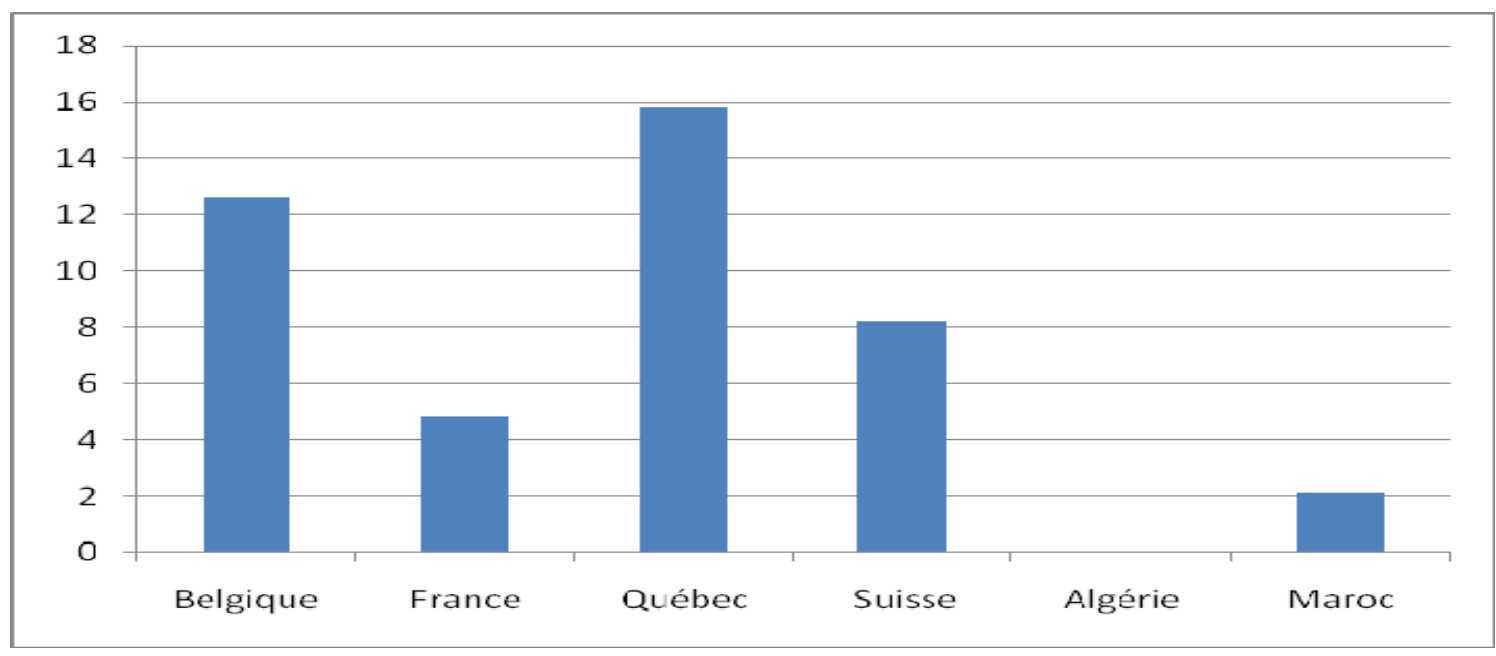

En effet, le test de Fisher indique un écart significatif des variances pour les comparaisons : Algérie/Belgique, Algérie/Québec, Algérie/Suisse, Belgique/France, Québec/France et Maroc/Québec $(p<.0001) ;$ Algérie/Suisse $(p=.0143)$; Belgique/Suisse $(p=.0237)$.

Il est intéressant, pour conclure sur ces tendances générales, d'observer le pourcentage de personnes enquêtées qu'elles représentent, ce que nous résumons dans le tableau suivant.

Tableau 3 : Pourcentage de sujets qui recourent aux propositions 3, 22, 6 et 19

\begin{tabular}{|l|c|c|}
\hline Proposition & \% de la pop. enquêtée & $\begin{array}{c}\text { \% pop. ayant répondu } \\
\text { à la question F }\end{array}$ \\
\hline $\begin{array}{l}3 \text { « simplifier l'orthographe, mais en conserver } \\
\text { 1'aspect symbolique » }\end{array}$ & 9,3 & 13,0 \\
\hline 22 «ne pas dévaloriser la langue » & 9,2 & 12,9 \\
\hline 6 « régulariser/rationaliser » & 11,1 & 15,5 \\
\hline 19 «être logique ». & 7,7 & 10,7 \\
\hline
\end{tabular}

Dans ces propositions, le point de vue des enseignants et des futurs enseignants rejoint le point de vue de spécialistes, tel Gruaz (2009) qui propose de "renforcer les régularités existantes », de " tenir compte de la fréquence », d' "éliminer les avatars historiques ", d'édicter des règles simples ne générant pas d'exceptions. Cependant, d'une part, comme nous l'avons vu au début, chacun a sa propre représentation des zones de difficultés, des zones où les exceptions gagneraient à être dissoutes dans la règle et, d'autre part, comme le souligne d'ailleurs Gruaz, le "pluri-système orthographique », pour reprendre le terme de Catach (1986), tisse des liens étroits entre les différents éléments qui le composent et il est très difficile de réformer un point, sans que d'autres soient touchés. Ainsi, des actions de pédagogie critique sur ce qui pourrait être réformable devraient être menées pour mettre au jour les questions posées et les réponses pouvant éventuellement y être apportées. 


\section{Conclusion}

Si l'orthographe reste pour une bonne partie des personnes interrogées un intouchable linguistique, pour d'autres, elle aurait tout de même besoin d'un « toilettage », et, pour d'autres encore, il conviendrait de régulariser en masse les kyrielles d'exceptions. Nos témoins s'entendent sur l'objectif d'une éventuelle réforme orthographique : elle devrait être de simplifier. Cet objectif de simplification est souvent assorti de réserves de différents ordres. Ainsi, des sujets convoquent la dimension linguistique, précisant que la simplification doit préserver le marquage et la finesse sémantiques et les fonctions morphographiques. Qu'elles soient associées à un refus catégorique de toute réforme ou à des restrictions quant à la portée d'éventuels aménagements, les réponses contiennent une troisième série d'opinions empreintes d'axiologie et d'idéologie, invoquant tantôt la "beauté », le "charme » ou la « richesse », fustigeant le spectre de la «dénaturation », de la perte d'identité ou de prestige, etc. Ces deux catégories de réserves (symbolique et idéologique) présentent à notre sens une zone commune et des frontières poreuses, et elles sont donc difficiles à distinguer clairement.

Dans les propositions que nous avons regroupées en deux autres blocs thématiques, les sujets se projettent dans l'élaboration et la mise en œuvre pour ainsi dire concrète de l'aménagement, en faisant des propositions concernant des modalités de décision (qui ? comment ?) et d'application, dont aucune ne parvient cependant à faire l'unanimité. Toutefois, un certain nombre de répondants évoquent l'importance d'assurer une large diffusion à toute mesure visant à aménager l'orthographe, notamment à travers l'enseignement.

De façon encore assez pragmatique, certains témoins énoncent également les qualités (clarté, cohérence, mesure ou, au contraire, radicalité) qu'une future réforme devrait réunir et des défauts ou erreurs dont il faudrait se garder.

Enfin une partie des témoins réaffirme à l'occasion de cette question qu'ils sont opposés à l'idée d'une réforme.

Ainsi, il nous faut souligner que la «bonne » réforme n'est pas consensuelle, mais qu'elle se dessine avec des contours assez dissemblables, telle que l'atteste, entre autres exemples, l'existence de positions quasi polaires tendant à mettre en avant des raisons d'ordre pragmatique d'un côté et d'ordre symbolique de l'autre. Précisons de plus que ces deux tendances ne sont pas exclusives l'une de l'autre : les segments de texte codés 3 correspondent en effet à des extraits qui combinent une volonté de simplifier tout en manifestant un attachement à certains aspects d'ordre symbolique.

Il apparait donc que l'orthographe reste bien une «institution» (Guion 1974) qui s'avère réinterprétée par des idéologies linguistiques différenciées amenant souvent à des prises de positions ambivalentes, voire paradoxales.

Un enseignement raisonné et critique de l'orthographe, de ses contraintes, de son histoire, de ses incohérences, de ses principes serait peut-être à même de faire évoluer les regards passionnés (cf. Closets 2009). Chaque scripteur de la langue française 
pourrait ainsi évaluer son outil d'écriture, armé d'arguments construits sur la connaissance objective de cet outil et non sur des appréciations intuitives, sensibles et fétichisées. Dans cette perspective, le souci de rendre l'orthographe du français fonctionnelle et accessible pourrait alors être autre chose, pour reprendre le mot de Chervel (2008), qu'un « divertissement de lettrés ».

\section{Bibliographie}

CAtach Nina, DuPREz Daniel et GruAz Claude (1986), L'orthographe françaiseTraité théorique et pratique. Paris : Nathan.

CHERVEL André (2008), "Réformer l'orthographe pour l'enseigner », entretien réalisé par Pascale Krémer. Le Monde 2, 6 décembre, 34-38.

Chevrot Jean-Pierre, Fontana Lucile, Freychet Laurent et Simon, Jean-Pascal (1994). «Comment les enseignants corrigent et évaluent les variations orthographiques ». Dans LuCCI Vincent et Millet Agnès (dir.), L'Orthographe de tous les jours. Enquête sur les pratiques orthographiques des Français. Paris : Champion, 139-190.

CLOSETS François de (2009), Zéro fautes, l'orthographe, une passion française. Paris : Mille et une nuits.

DAVID Jacques (2010), «Place et importance de l'orthographe à l'école et dans la formation des enseignants ». Dans GRUAZ Claude et JACQUET-Pfau Chirstine( dir.), Autour du mot : pratiques et compétences. Limoges : Lambert-Lucas, 107129.

GROUPE RO (2011), Faut-il réformer l'orthographe? Craintes et attentes des francophones. Français et société, $\mathrm{n}^{\circ}$ 21. Bruxelles: Service de la langue française ; Fernelmont : EME.

GRUAZ Claude (2009), «Vers une rationalisation de l'orthographe française ». Dans Dister Anne et al., Penser l'orthographe de demain. Paris : CILF, 75-86.

GuION Jean (1974), L'institution orthographe. Paris : Le Centurion, coll. Paidoguides.

Moreau Marie-Louise \& Dister Anne (2012, coord.) Réforme(s) de l'orthographe Craintes, attentes et réactions des citoyens - Volonté et stratégies politiques, Glottopol 19.

SILVERSTEIN Michael (1979), «Language structure and linguistic ideology ». Dans Clyne Paul R., HANCKS William F. \& HofBauer Carol L. (éds), The elements: A parasession on linguistic units and levels. Chicago : Chicago Linguistic Society, 193-247.

WoOlARD Kathryn A. (1998), «Language ideology as a field of inquiry ». Dans SCHIEFFElin Bambi B., WoOlard Kathryn A. et Kroskrity Paul V. (éds), Language ideologies. practice and theory. Oxford et New York : Oxford University Press, 3-47. 